\title{
Quantum Mechanics on a Curved Snyder Space
}

\author{
Salvatore Mignemi ${ }^{1,2}$ and Rina Štrajn ${ }^{1,2}$ \\ ${ }^{1}$ Dipartimento di Matematica e Informatica, Università di Cagliari, Viale Merello 92, 09123 Cagliari, Italy \\ ${ }^{2}$ INFN, Sezione di Cagliari, Cittadella Universitaria, 09042 Monserrato, Italy
}

Correspondence should be addressed to Salvatore Mignemi; smignemi@unica.it

Received 17 February 2016; Accepted 12 May 2016

Academic Editor: Antonio Ereditato

Copyright (C) 2016 S. Mignemi and R. Štrajn. This is an open access article distributed under the Creative Commons Attribution License, which permits unrestricted use, distribution, and reproduction in any medium, provided the original work is properly cited. The publication of this article was funded by SCOAP $^{3}$.

\begin{abstract}
We study the representations of the three-dimensional Euclidean Snyder-de Sitter algebra. This algebra generates the symmetries of a model admitting two fundamental scales (Planck mass and cosmological constant) and is invariant under the Born reciprocity for exchange of positions and momenta. Its representations can be obtained starting from those of the Snyder algebra and exploiting the geometrical properties of the phase space that can be identified with a Grassmannian manifold. Both the position and momentum operators turn out to have a discrete spectrum.
\end{abstract}

\section{Introduction}

The Snyder-de Sitter (SdS) model, or triply special relativity [1], was introduced as a generalization of the Snyder model [2] to a curved background.

The Snyder model has been the first example of noncommutative geometry proposed in the literature and is based on a deformation of the Heisenberg algebra by a fundamental invariant scale $\beta$ with dimension of inverse energy. It was introduced in the hope of achieving a regularization of field theory through the new scale, whose presence, contrary to what one may expect, does not affect the Lorentz invariance but only deforms the translation symmetry [3].

The Snyder model can also be considered as an example of doubly special relativity, namely, a theory where a new fundamental scale is introduced by deforming special relativity $[4,5]$. Models of this sort can be described in terms of a curved momentum space [6], which in the Snyder case is a 3 -sphere $S^{3}$. In general, the nontrivial geometry of the momentum space entails some remarkable consequences on the definition of locality that loses its absolute meaning and becomes observer dependent, suggesting the possibility that locality is a relative property [7].

The properties of the Snyder space and its dynamics, both in the nonrelativistic and relativistic version, have been investigated in several papers and in various contexts, both classical and quantum [8-26]. In particular, it was shown that space is discretized [9] and deformed Heisenberg uncertainty relations hold, implying a lower bound on measurable length [8]. Both classical and quantum dynamics are modified with respect to the standard results, with deviations of order $\beta^{2} E^{2}$, $E$ being the energy of the system [8].

The extension of the Snyder model to a spacetime background of constant curvature was proposed in [1] (see also [27] for a different approach). This generalization was motivated by the necessity of including the cosmological constant $\Lambda \sim \alpha^{2}$ among the bare parameters of a theory of quantum gravity [1]. In this way, one introduces a third fundamental constant besides the speed of light $c$ and the Snyder parameter $\beta$, which explains the name of triply special relativity originally given to the theory. The most relevant feature of this generalization is its duality for the interchange between positions and momenta that realizes Born reciprocity principle [28]. The properties of SdS space were studied by many authors [29-37], mainly in its nonrelativistic version: it turns out that both positions and momenta have discrete spectra, and a minimal momentum occurs besides minimal length. More general theories with curved momentum space on a curved spacetime background have also been studied from the point of view of relative locality in $[38,39]$, especially in relation with cosmology and geodesic motion. 
In this paper, we attempt to generalize the results on the algebraic structure of the three-dimensional nonrelativistic Snyder model, investigated in [9], to the case of a curved background. Although the nonrelativistic limit is physically less interesting than the relativistic theory, it can shed some light on the structure of the theory and serve as a first step in the construction of a quantum field theory. Of course, in this limit only two fundamental constants, $\alpha$ and $\beta$, are left.

In the SdS case, the algebraic structure is less useful than in the Snyder case, because for SdS the spectrum of the position operator cannot be derived directly from the algebraic structure of the theory. It is however possible to find a relation between the representations of Snyder and of SdS, which enables one to find analytically the spectrum of the square of the position and momentum operators. Because of the Born reciprocity these spectra are essentially identical.

1.1. The SdS Model. The nonrelativistic SdS algebra $\mathscr{A}$ depends on two parameters $\alpha$ and $\beta$ and contains the usual generators of rotations $\widehat{L}_{k}=(1 / 2) \epsilon_{i j k} \widehat{L}_{i j}(i, j=1, \ldots, 3)$, with their standard action on the position and momentum operators $\widehat{x}_{i}$ and $\widehat{p}_{i}$ :

$$
\begin{aligned}
& {\left[\widehat{L}_{i}, \widehat{L}_{j}\right]=i \epsilon_{i j k} \widehat{L}_{k},} \\
& {\left[\widehat{L}_{i}, \widehat{x}_{j}\right]=-i \epsilon_{i j k} \widehat{x}_{k},} \\
& {\left[\widehat{L}_{i}, \widehat{p}_{j}\right]=-i \epsilon_{i j k} \widehat{p}_{k} .}
\end{aligned}
$$

The commutation relations of $\hat{x}_{i}$ and $\widehat{p}_{i}$ satisfy instead a deformation of the Heisenberg algebra

$$
\begin{aligned}
& {\left[\widehat{x}_{i}, \widehat{x}_{j}\right]=i \beta^{2} \epsilon_{i j k} \widehat{L}_{k},} \\
& {\left[\widehat{p}_{i}, \widehat{p}_{j}\right]=i \alpha^{2} \epsilon_{i j k} \widehat{L}_{k},} \\
& {\left[\widehat{x}_{i}, \widehat{p}_{j}\right]} \\
& \quad=i\left[\delta_{i j}+\alpha^{2} \widehat{x}_{i} \widehat{x}_{j}+\beta^{2} \widehat{p}_{j} \widehat{p}_{i}+\alpha \beta\left(\hat{x}_{j} \widehat{p}_{i}+\widehat{p}_{i} \hat{x}_{j}\right)\right],
\end{aligned}
$$

where $\widehat{L}_{i j}=(1 / 2)\left(\widehat{x}_{i} \widehat{p}_{j}+\widehat{p}_{j} \widehat{x}_{i}-\widehat{x}_{j} \widehat{p}_{j}-\widehat{p}_{i} \widehat{x}_{j}\right)$.

For special values of the parameters, $\mathscr{A}$ reduces to the nonrelativistic Snyder algebra $(\alpha=0)$ or the algebra of isometries of $S^{3}$ in Beltrami coordinates $(\beta=0)$. It is possible to define also a noncompact version of the algebra, by analytic continuation to imaginary values of $\alpha$ and $\beta$, with rather different properties [29], but we will not discuss it here. As mentioned above, the algebra is invariant for $\alpha x_{\mu} \leftrightarrow \beta p_{\mu}$. More generally, it is invariant for rotations in the phase space, $\alpha x_{i} \rightarrow \alpha x_{i} \cos \theta+\beta p_{i} \sin \theta, \beta p_{i} \rightarrow-\alpha x_{i} \sin \theta+\beta p_{i} \cos \theta$. Of course, this symmetry holds also in standard quantum mechanics, with $\alpha=\beta=1$.

The SdS algebra can be considered as a nonlinear realization of a model proposed by Yang [40], which differs from SdS only in the assumption of a standard Heisenberg algebra for positions and momenta, $\left[\widehat{x}_{i}, \widehat{p}_{j}\right]=i \widehat{K} \delta_{i j}, \widehat{K}$ being a central charge for the rotation group, satisfying $\left[\widehat{K}, \widehat{x}_{i}\right]=i \alpha^{2} \widehat{p}_{i}$, $\left[\widehat{K}, \widehat{p}_{i}\right]=-i \beta^{2} \widehat{x}_{i}$. With the identifications $\widehat{L}_{i j}=\widehat{J}_{i j}, \alpha \widehat{x}_{i}=\widehat{J}_{4 i}$, $\beta \widehat{p}_{i}=\widehat{J}_{5 i}, \widehat{K}=\widehat{J}_{45}$, the Yang model reproduces an so(5) algebra with generators $\widehat{J}_{\mu \nu},(\mu, \nu=1, \ldots, 5)$.

Also the 3-dimensional nonrelativistic SdS model enjoys an $\mathrm{SO}(5)$ symmetry. In fact, its phase space can be realized on the six-dimensional Grassmannian coset space $\operatorname{Gr}(3,5)=$ $\mathrm{SO}(5) / \mathrm{SO}(3) \times \mathrm{SO}(2)$, with $\mathrm{SO}(3)$ generated by the $J_{i j}$ and $\mathrm{SO}(2)$ by $J_{45}$.

The space $\operatorname{Gr}(3,5)$ can be parametrized by homogeneous coordinates $x_{\mu}$ and $p_{\mu}$, that satisfy the constraints [41]:

$$
\begin{aligned}
& \alpha^{2} x_{\mu}^{2}=1, \\
& \beta^{2} p_{\mu}^{2}=1, \\
& x_{\mu} p_{\mu}=0 .
\end{aligned}
$$

This parametrization associates a one-parameter set of matrices to each coset. One can then identify the variables $x_{\mu}$ and $p_{\mu}$ with canonical coordinates of a ten-dimensional phase space and hence reduce it to a six-dimensional phase space parametrized by $x_{i}$ and $p_{i}$ by eliminating the constraints (3), using the Dirac formalism [42]. In order to obtain a one-toone parametrization, one has however to impose a further constraint on the parameters $x_{4}, x_{5}, p_{4}, p_{5}$. This is also required by the Dirac formalism since the constraints (3) split into one first class and two second class constraints [32]. Unfortunately, not every constraint leads to the SdS algebra, and one has therefore to choose a suitable gauge [32]. In particular, the choice $\alpha x_{5}+\beta p_{5}=0$ yields the algebra (2).

\section{The Nonrelativistic Snyder Algebra}

In this section we review some results on the representation of the Snyder algebra from $[8,9]$ that will be useful in the following discussion.

The Snyder algebra is the limit of the SdS algebra (1)-(2) for $\alpha \rightarrow 0$. It contains an so(4) subalgebra of so(5) generated by $\widehat{X}_{i}=\widehat{J}_{4 i}$ and $\widehat{L}_{i}=(1 / 2) \epsilon_{i j k} \widehat{J}_{j k}$, while the momentum space is realized as the coset space $S^{3}=\mathrm{SO}(4) / \mathrm{SO}(3)$.

The representations of the so(4) algebra can be labeled by the eigenvalues of $\widehat{A}_{i}^{2}, \widehat{L}_{i}^{2}$, and $\widehat{L}_{3}$, where $\widehat{A}_{i}$ is the operator $(1 / 2)\left(\widehat{L}_{i}+\beta^{-1} \widehat{X}_{i}\right)$, so that $\widehat{X}_{i}^{2}=\beta^{2}\left(4 \widehat{A}_{i}^{2}-\widehat{L}_{i}^{2}\right)$ :

$$
\begin{aligned}
& \widehat{L}_{i}^{2}|j, l, m\rangle=l(l+1)|j, l, m\rangle, \\
& \widehat{L}_{3}|j, l, m\rangle=m|j, l, m\rangle, \\
& \widehat{X}_{i}^{2}|j, l, m\rangle=\beta^{2}[4 j(j+1)-l(l+1)]|j, l, m\rangle,
\end{aligned}
$$

with $0 \leq l \leq 2 j, j(j+1)$ being the eigenvalue of $\widehat{A}_{i}^{2}$. The eigenvalues of $\widehat{X}_{i}^{2}$ have degeneration $2 l+1$.

The momentum space can be realized as a 3 -sphere, obtained by imposing the constraint $P_{i}^{2}+P_{4}^{2}=1 / \beta^{2}$ on a four-vector $P$. This can be shown algebraically, as in [9], or also from a Dirac reduction of the phase space [11]. In the following, we will mainly concentrate on the operators $\widehat{X}_{i}^{2}$ and $\widehat{P}_{i}^{2}$ and investigate their spectra. 
2.1. The Representation I. One can define several different representations of the Snyder algebra on a Hilbert space. Usually they are given in momentum representation. In $[9,10]$ it was realized by operators $\widehat{P}_{i}$ and $\widehat{X}_{i}$ defined as

$$
\begin{aligned}
& \widehat{P}_{i}=P_{i} \\
& \widehat{X}_{i}=i \frac{\partial}{\partial P_{i}}+i \beta^{2} P_{i}\left(P_{j} \frac{\partial}{\partial P_{j}}+\mu\right) \\
& \widehat{L}_{i}=-i \epsilon_{i j k} P_{j} \frac{\partial}{\partial P_{k}}
\end{aligned}
$$

which act on functions $\psi\left(P_{i}\right)$ of the Hilbert space, with $\mu$ an arbitrary real parameter and $-\infty<P_{i}<\infty$. The operators are symmetric for the measure

$$
\frac{d^{3} P}{\left(1+\beta^{2} P_{i}^{2}\right)^{2-\mu}}
$$

provided that the functions $\psi\left(P_{i}\right)$ go to infinity like $\left(1 / \sqrt{P_{i}^{2}}\right)^{\mu-1 / 2}$.

In this representation the operator $\widehat{X}_{i}^{2}$ reads

$$
\begin{aligned}
\widehat{X}_{i}^{2} & \\
= & -\left(1+\beta^{2} P_{\rho}^{2}\right)^{2}\left(\frac{\partial^{2}}{\partial P_{\rho}^{2}}+\frac{2}{P_{\rho}} \frac{\partial}{\partial P_{\rho}}\right) \\
& -\mu \beta^{2}\left[2\left(1+\beta^{2} P_{\rho}^{2}\right) P_{\rho} \frac{\partial}{\partial P_{\rho}}+(1+\mu) \beta^{2} P_{\rho}^{2}+3\right] \\
& +\frac{\widehat{L}_{i}^{2}}{P_{\rho}^{2}} .
\end{aligned}
$$

For $\mu=0$, the equation $\widehat{X}_{i}^{2} \phi=X_{i}^{2} \phi$ has eigenfunctions [9]

$$
\phi_{n l m}=\text { const. } \times \sin ^{l} \chi C_{n}^{(l+1)}(\cos \chi) Y_{m}^{l}\left(P_{\theta}, P_{\varphi}\right),
$$

where we have used polar coordinates $P_{\rho}, P_{\theta}, P_{\varphi}$ for $P_{i}$, and $\chi=\arctan \beta P_{\rho}$. The functions $C_{n}^{(a)}$ are Gegenbauer polynomials with $n$ a nonnegative integer parameter and $Y_{m}^{l}\left(P_{\theta}, P_{\varphi}\right)$ spherical harmonics.

It is easy to see that if $\mu \neq 0$, the eigenfunctions (8) are simply multiplied by $\cos ^{\mu} \chi$. The eigenvalues are of course independent of $\mu$ and read

$$
X_{i}^{2}=\beta^{2}\left(n^{2}+2 n l+2 n+l\right),
$$

with $0 \leq l \leq n$. They can easily be identified with (4) by setting $n=2 j-l$.

The operator $\widehat{P}_{i}^{2}=\widehat{P}_{\rho}^{2}$ is trivial and its spectrum extends to the real positive line.
2.2. The Representation II. An alternative representation is obtained [8] by defining

$$
\begin{aligned}
& \widehat{P}_{i}=\frac{P_{i}}{\sqrt{1-\beta^{2} P_{k}^{2}}}, \\
& \widehat{X}_{i}=i \sqrt{1-\beta^{2} P_{k}^{2}} \frac{\partial}{\partial P_{i}},
\end{aligned}
$$

with $P_{k}^{2}<1 / \beta^{2}$. In this representation the operators are symmetric for the measure

$$
\frac{d^{3} P}{\sqrt{1-\beta^{2} P_{k}^{2}}}
$$

and the operator $\widehat{X}_{i}^{2}$ reads

$$
\begin{aligned}
\widehat{X}_{i}^{2}= & -\left(1-\beta^{2} P_{\rho}^{2}\right) \frac{\partial^{2}}{\partial P_{\rho}^{2}}-\frac{2-3 \beta^{2} P_{\rho}^{2}}{P_{\rho}} \frac{\partial}{\partial P_{\rho}} \\
& +\frac{\left(1-\beta^{2} P_{\rho}^{2}\right) \widehat{L}_{i}^{2}}{P_{\rho}^{2}} .
\end{aligned}
$$

It has eigenfunctions

$$
\begin{aligned}
\phi_{q l m}= & \text { const. } \\
& \times \sin ^{l} \eta \cos \eta P_{q}^{(1 / 2, l+1 / 2)}(\cos 2 \eta) Y_{m}^{l}\left(P_{\theta}, P_{\varphi}\right),
\end{aligned}
$$

where $\eta=\arcsin \beta P_{\rho}$ and $P_{q}^{(a, b)}$ are Jacobi polynomials with $q$ a nonnegative integer. The eigenvalues are given by $\beta^{2}[(2 q+$ $\left.l+2)^{2}-l(l+1)-1\right]$. Taking $q=(n-1) / 2$, one recovers the eigenvalues (9).

\section{The Nonrelativistic SdS Algebra}

The representations of the operators $\widehat{x}_{i}$ and $\widehat{p}_{i}$ that satisfy the SdS algebra can be obtained from the operators $\widehat{X}_{i}$ and $\widehat{P}_{i}$ of the Snyder algebra by taking the linear combinations [29]

$$
\begin{aligned}
& \widehat{x}_{i}=\widehat{X}_{i}+\lambda \frac{\beta}{\alpha} \widehat{P}_{i}, \\
& \widehat{P}_{i}=(1-\lambda) \widehat{P}_{i}-\frac{\alpha}{\beta} \widehat{X}_{i},
\end{aligned}
$$

with inverse

$$
\begin{aligned}
& \widehat{P}_{i}=\widehat{p}_{i}+\frac{\alpha}{\beta} \widehat{x}_{i}, \\
& \widehat{X}_{i}=(1-\lambda) \widehat{x}_{i}-\lambda \frac{\beta}{\alpha} \widehat{p}_{i},
\end{aligned}
$$

where $\lambda$ is a free parameter. Representations with different values of $\lambda$ are related by unitary transformations [29]; therefore in the following we will consider only the case $\lambda=0$.

The relation between Snyder and SdS representations can be understood by considering the embedding of $S^{3}$ into 
$\mathrm{Gr}(3,5)$, corresponding to the branching $\mathrm{SO}(5) \rightarrow \mathrm{SO}(4)$. We recall that the vectors of $\operatorname{Gr}(3,5)$ satisfy the constraints (3), while those of $S^{3}$ satisfy $\beta^{2}\left(P_{i}^{2}+P_{4}^{2}\right)=1$. Taking into account the SdS gauge constraint $\alpha x_{5}+\beta p_{5}=0$, it is easy to see that the combination $P_{\mu}=p_{\mu}+(\alpha / \beta) x_{\mu}$ defined as in (15) satisfies the same constraint as the vectors of $\mathrm{SO}(4) / \mathrm{SO}(3)$ and then transforms as the Snyder momentum.

3.1. The Momentum Representation I. Setting $\lambda=0$, from (14) and (5) one obtains the representation

$$
\begin{aligned}
& \widehat{x}_{i}=i \frac{\partial}{\partial P_{i}}+i \beta^{2} P_{i}\left(P_{j} \frac{\partial}{\partial P_{j}}+\mu\right), \\
& \widehat{P}_{i}=P_{i}-i \frac{\alpha}{\beta}\left[\frac{\partial}{\partial P_{i}}+\beta^{2} P_{i}\left(P_{j} \frac{\partial}{\partial P_{j}}+\mu\right)\right] .
\end{aligned}
$$

As for the Snyder model, the eigenfunctions can be written in terms of $\widehat{x}_{i}^{2}, \widehat{L}^{2}$, and $\widehat{L}_{3}$.

Clearly, $\widehat{x}_{i}^{2}=\widehat{X}_{i}^{2}$, and hence the equation

$$
\widehat{x}_{i}^{2} \psi=x_{i}^{2} \psi
$$

has the same eigenfunctions (8) and eigenvalues (9) as in the Snyder model.

The calculation of the operator $\widehat{p}_{i}^{2}$ is a bit more involved. From (14) one has, for $\lambda=0$,

$$
\widehat{P}_{i}^{2}=\widehat{P}_{i}^{2}-\frac{\alpha}{\beta}\left(\widehat{X}_{i} \widehat{P}_{i}+\widehat{P}_{i} \widehat{X}_{i}\right)+\frac{\alpha^{2}}{\beta^{2}} \widehat{X}_{i}^{2} .
$$

In the representation of Section 2.2,

$$
\begin{aligned}
\widehat{X}_{i} \widehat{P}_{i}+\widehat{P}_{i} \widehat{X}_{i}= & 2 i\left(1+\beta^{2} P_{\rho}^{2}\right) P_{\rho} \frac{\partial}{\partial P_{\rho}}+3 i \\
& +i \beta^{2}(1+2 \mu) P_{\rho}^{2} .
\end{aligned}
$$

From (7) and (19) it follows then that

$$
\begin{aligned}
\widehat{p}_{i}^{2} & =-\frac{\alpha^{2}}{\beta^{2}}\left[\left(1+\beta^{2} P_{\rho}^{2}\right)^{2} \frac{\partial^{2}}{\partial P_{\rho}^{2}}\right. \\
& \left.+\left(1+\beta^{2} P_{\rho}^{2}\right)\left(1+\beta^{2} P_{\rho}^{2}+i \frac{\beta}{\alpha} P_{\rho}^{2}\right) \frac{2}{P_{\rho}} \frac{\partial}{\partial P_{\rho}}-\frac{\widehat{L}_{i}^{2}}{P_{\rho}^{2}}\right] \\
& -\frac{\alpha}{\beta}\left[3 i+\left(i \beta^{2}-\frac{\beta}{\alpha}\right) P_{\rho}^{2}\right] \\
& +\mu \alpha^{2}\left[2\left(1+\beta^{2} P_{\rho}^{2}\right) P_{\rho} \frac{\partial}{\partial P_{\rho}}+(1+\mu) \beta^{2} P_{\rho}^{2}+3\right. \\
& \left.-2 i \frac{\beta}{\alpha} P_{\rho}^{2}\right] .
\end{aligned}
$$

As for the Snyder model, it is straightforward to check that the solutions with $\mu \neq 0$ can simply be obtained by multiplying those with vanishing $\mu$ by $\cos ^{\mu} \chi$, so we consider only the case $\mu=0$. Then, the solutions of the eigenvalue equation $\widehat{p}_{i}^{2} \phi=p_{i}^{2} \phi$ can be deduced from those of (17) by noting that the substitution $\phi=\left(1+\beta^{2} P_{\rho}^{2}\right)^{-i / 2 \alpha \beta} \psi$ brings the equation to the same form as (7), with $X_{i}^{2} \rightarrow\left(\beta^{2} / \alpha^{2}\right) p_{i}^{2}$, and hence its eigenfunctions differ only by a phase from those of $\widehat{x}_{i}^{2}$ :

$$
\begin{aligned}
\phi_{n l m}= & \text { const. } \\
& \times \sin ^{l} \chi \cos ^{i / \alpha \beta} \chi C_{n}^{(l+1)}(\cos \chi) Y_{m}^{l}\left(P_{\theta}, P_{\varphi}\right) .
\end{aligned}
$$

The operators $\widehat{x}_{i}^{2}$ and $\widehat{p}_{i}^{2}$ are therefore related by a unitary transformation, and the eigenvalues of $\widehat{p}_{i}^{2}$ are the same as those of $\hat{x}_{i}^{2}$, except for a multiplicative constant:

$$
p_{i}^{2}=\alpha^{2}\left(n^{2}+2 n l+2 n+l\right)
$$

This could have been predicted on the ground of the duality between $\widehat{x}_{i}$ and $\widehat{p}_{i}$. It follows that in the SdS model also the eigenvalues of the momentum square (and hence of the energy) are quantized and that they do not depend on $\beta$.

3.2. The Momentum Representation II. Also for SdS one can adopt the alternative representation of Section 2.2 [29]. Starting from (10), one obtains, for $\lambda=0$,

$$
\begin{aligned}
& \hat{x}_{i}=i \sqrt{1-\beta^{2} P_{k}^{2}} \frac{\partial}{\partial P_{i}}, \\
& \widehat{p}_{i}=\frac{P_{i}}{\sqrt{1-\beta^{2} P_{k}^{2}}}-i \frac{\alpha}{\beta} \sqrt{1-\beta^{2} P_{k}^{2}} \frac{\partial}{\partial P_{i}} .
\end{aligned}
$$

As before, for $\lambda=0$, the operator $\widehat{x}_{i}^{2}$ coincides with $\widehat{X}_{i}^{2}$ and its eigenfunctions and eigenvalues are given, respectively, by (13) and (9).

The operator $\widehat{p}_{i}^{2}$ reads instead

$$
\begin{aligned}
\widehat{p}_{i}^{2} & =-\frac{\alpha^{2}}{\beta^{2}}\left[\left(1-\beta^{2} P_{\rho}^{2}\right) \frac{\partial^{2}}{\partial P_{\rho}^{2}}\right. \\
& \left.+\frac{2-\left(3 \beta^{2}+2 i(\beta / \alpha)\right) P_{\rho}^{2}}{P_{\rho}} \frac{\partial}{\partial P_{\rho}}\right] \\
& -\frac{(1+2 i \alpha \beta) P_{\rho}^{2}+3 i(\alpha / \beta)}{\left(1-\beta^{2} P_{\rho}^{2}\right)}+\frac{\alpha^{2} \widehat{L}^{2}}{\beta^{2} P_{\rho}^{2}} .
\end{aligned}
$$

This result had been obtained in [29] for a slightly different operator.

In analogy with the calculations done in the previous section, the eigenvalue equation for $\widehat{p}_{i}^{2}$ can be reduced to the form (12) by introducing a function $\psi$ such that $\phi=(1-$ $\left.\beta^{2} P_{\rho}^{2}\right)^{i / 2 \alpha \beta} \psi$. The solution is therefore

$$
\phi_{\text {qml }}=\text { const. } \times \sin ^{l} \eta \cos ^{1+i / 2 \alpha \beta} \eta P_{q}^{(l+1 / 2,1 / 2)}(\cos 2 \eta) Y_{m}^{l}\left(P_{\theta}, P_{\varphi}\right),
$$

with $\eta=\arcsin \beta P_{\rho}$. As in the Snyder case, taking $q=(n+$ $1) / 2$, one recovers the eigenvalues (22). 
3.3. The Position Representations. The duality of the SdS algebra for interchange of $\widehat{x}_{i}$ with $\widehat{p}_{i}$ permits to define position representations by simply exchanging the roles of the phase space coordinates. Alternatively, such representations can be obtained starting from those of the symmetries of $S^{3}$ in Beltrami coordinates and using transformations analogous to (14).

From (16) and (23) one obtains in this way the action of the momentum and position operators on the Hilbert space of functions of $X_{i}$. We report them in the case $\lambda=0$, where they read, respectively,

$$
\begin{aligned}
& \widehat{p}_{i}=i \frac{\partial}{\partial X_{i}}+i \alpha^{2} X_{i}\left(X_{k} \frac{\partial}{\partial X_{k}}+\mu\right) \\
& \widehat{x}_{i}=X_{i}-i \frac{\beta}{\alpha}\left[\frac{\partial}{\partial X_{i}}+\alpha^{2} X_{i}\left(X_{k} \frac{\partial}{\partial X_{k}}+\mu\right)\right], \\
& \widehat{p}_{i}=i \sqrt{1-\alpha^{2} X_{k}^{2}} \frac{\partial}{\partial X_{i}} \\
& \widehat{x}_{i}=\frac{X_{i}}{\sqrt{1-\alpha^{2} X_{k}^{2}}}-i \frac{\beta}{\alpha} \sqrt{1-\alpha^{2} X_{k}^{2}} \frac{\partial}{\partial X_{i}} .
\end{aligned}
$$

Position representations can be useful in some problems, like the hydrogen atom, where the potential is a nontrivial function of $X_{i}$.

\section{Conclusions}

We have investigated some properties of the nonrelativistic SdS algebra and in particular its Hilbert space representations. This algebra is notable because it generates deformed commutation relations without breaking the Lorentz invariance. Since position and momentum are related by a duality, their operators have identical spectra, except for a multiplicative constant, and both are discrete due to the compactness of the algebra. Of course many other unitary equivalent representations exist besides the ones considered in this paper that lead to the same physical results.

Our discussion may be useful for the study of nontrivial systems in SdS space. At present, only the free particle and the harmonic oscillator have been discussed [29]. An interesting system to investigate would be the hydrogen atom. However, preliminary calculations seem to indicate that it leads to third-order differential equations that are difficult to study.

The results of this paper may also be extended without much effort to the case of negative coupling constants in the algebra (2). In that case the algebra is no longer compact and the spectra of positions and momenta are expected to be continuous [29].

Another interesting development would be the investigation of the model from the point of view of relative locality, starting from the discussion of theories with nontrivial momentum space geometry in curved spacetimes initiated in $[38,39]$.

Finally, as suggested in [9] in the discussion of the flat space limit, our results may also be employed for the construction of a quantum field theory on curved Snyder space, by exploiting the lattice-like structure that has emerged from our investigation.

\section{Competing Interests}

The authors declare that they have no competing interests.

\section{References}

[1] J. Kowalski-Glikman and L. Smolin, "Triply special relativity," Physical Review D, vol. 70, no. 6, Article ID 065020, 6 pages, 2004.

[2] H. S. Snyder, “Quantized space-time," Physical Review, vol. 71, no. 1, pp. 38-41, 1947.

[3] S. Mignemi, "Doubly special relativity and translation invariance," Physics Letters B, vol. 672, no. 2, pp. 186-189, 2009.

[4] G. Amelino-Camelia, "Testable scenario for relativity with minimum length," Physics Letters B, vol. 510, no. 1-4, pp. 255263, 2001.

[5] G. Amelino-Camelia, "Relativity in spacetimes with short-distance structure governed by an observer-independent (Planckian) length scale," International Journal of Modern Physics D: Gravitation, Astrophysics, Cosmology, vol. 11, no. 1, pp. 35-59, 2002.

[6] J. Kowalski-Glikman and S. Nowak, "Non-commutative spacetime of doubly special relativity theories," International Journal of Modern Physics D, vol. 12, no. 2, pp. 299-315, 2003.

[7] G. Amelino-Camelia, L. Freidel, J. Kowalski-Glikman, and L. Smolin, "Relative locality and the soccer ball problem," Physical Review D-Particles, Fields, vol. 84, no. 8, Article ID 087702, 2011.

[8] S. Mignemi, "Classical and quantum mechanics of the nonrelativistic Snyder model," Physical Review D, vol. 84, no. 2, Article ID 025021, 11 pages, 2011.

[9] L. Lu and A. Stern, "Snyder space revisited," Nuclear Physics B, vol. 854, no. 3, pp. 894-912, 2012.

[10] E. J. Hellund and K. Tanaka, "Quantized space-time," APS Journals Archive, vol. 94, no. 1, p. 192, 1954.

[11] F. Girelli, T. Konopka, J. Kowalski-Glikman, and E. R. Livine, "Free particle in deformed special relativity," Physical Review D, vol. 73, no. 4, Article ID 045009, 14 pages, 2006.

[12] J. M. Romero and A. Zamora, "Snyder noncommutative spacetime from two-time physics," Physical Review D, vol. 70, no. 10, Article ID 105006, 5 pages, 2004.

[13] J. M. Romero, J. D. Vergara, and J. A. Santiago, "Noncommutative spaces, the quantum of time, and Lorentz symmetry," Physical Review D, vol. 75, no. 6, Article ID 065008, 7 pages, 2007.

[14] R. Banerjee, S. Kulkarni, and S. Samanta, "Deformed symmetry in snyder space and relativistic particle dynamics," Journal of High Energy Physics, vol. 2006, no. 5, p. 77, 2006.

[15] M. V. Battisti and S. Meljanac, "Modification of Heisenberg uncertainty relations in noncommutative Snyder space-time geometry," Physical Review D, vol. 79, no. 6, Article ID 067505, 2009.

[16] M. V. Battisti and S. Meljanac, "Scalar field theory on noncommutative Snyder spacetime," Physical Review D, vol. 82, no. 2, Article ID 024028, 2010.

[17] C. Leiva, "Harmonic oscillator in Snyder space: the classical case and the quantum case," Pramana, vol. 74, no. 2, pp. 169-175, 2010. 
[18] C. Leiva, J. Saavedra, and J. R. Villanueva, "The kepler problem in the snyder space," Pramana, vol. 80, no. 6, pp. 945-951, 2013.

[19] F. Girelli and E. R. Livine, "Scalar field theory in Snyder spacetime: alternatives," Journal of High Energy Physics, vol. 2011, article 132, 2011.

[20] P. G. Castro, R. Kullock, and F. Toppan, "Snyder noncommutativity and pseudo-Hermitian HAMiltonians from a Jordanian twist," Journal of Mathematical Physics, vol. 52, no. 6, 062105, 9 pages, 2011.

[21] L. Lu and A. Stern, "Particle dynamics on Snyder space," Nuclear Physics B, vol. 860, no. 1, pp. 186-205, 2012.

[22] S. Pramanik and S. Ghosh, "GUP-based and Snyder noncommutative algebras, relativistic particle models, deformed symmetries and interaction: a unified approach," International Journal of Modern Physics A, vol. 28, no. 27, Article ID 1350131, 15 pages, 2013.

[23] S. Pramanik, S. Ghosh, and P. Pal, "Conformal invariance in noncommutative geometry and mutually interacting Snyder particles," Physical Review D, vol. 90, Article ID 105027, 2014.

[24] J. M. Lorenzi, R. Montemayor, and L. F. Urrutia, "A five dimensional perspective on many particles in the Snyder basis of double special relativity," in Proceedings of the 2nd Cinvestavunam Symposium on High Energy Physics, Particles and Neutrinos in an Astrophysical Context: in Honor of Juan Carlos D'olivo, vol. 1540 of AIP Conference Proceedings, p. 95, Mexico City, Mexico, October 2012.

[25] M. A. Gorji, K. Nozari, and B. Vakili, "Spacetime singularity resolution in Snyder noncommutative space," Physical Review D, vol. 89, no. 8, Article ID 084072, 2014.

[26] G. Amelino-Camelia and V. Astuti, "Misleading inferences from discretization of empty spacetime: snyder-noncommutativity case study," International Journal of Modern Physics. D, vol. 24, no. 10, Article ID 1550073, 2015.

[27] S. Mignemi, "Doubly special relativity in de Sitter spacetime," Annalen der Physik, vol. 522, no. 12, pp. 924-940, 2010.

[28] M. Born, "Reciprocity theory of elementary particles," Reviews of Modern Physics, vol. 21, no. 3, pp. 463-473, 1949.

[29] S. Mignemi, "Classical and quantum mechanics of the nonrelativistic Snyder model in curved space," Classical and Quantum Gravity, vol. 29, no. 21, 215019, 19 pages, 2012.

[30] C. Chryssomakolos and E. Okon, "Linear form of 3-scale special relativity algebra and the relevance of stability," International Journal of Modern Physics D, vol. 13, no. 9, p. 1817, 2004.

[31] H. G. Guo, C. G. Huang, and H. T. Wu, "Yang's model as triply special relativity and the Snyder's model-de Sitter special relativity duality," Physics Letters B, vol. 663, no. 3, pp. 270-274, 2008.

[32] S. Mignemi, "The Snyder-de Sitter model from six dimensions," Classical and Quantum Gravity, vol. 26, no. 24, Article ID 245020, 9 pages, 2009.

[33] M. C. Carrisi and S. Mignemi, "Snyder-de Sitter model from two-time physics," Physical Review D, vol. 82, no. 10, Article ID 105031, 5 pages, 2010.

[34] R. Banerjee, K. Kumar, and D. Roychowdhury, "Symmetries of Snyder-de SITter space and relativistic particle dynamics," Journal of High Energy Physics, vol. 2011, article 60, 2011.

[35] B. Ivetić, S. Meljanac, and S. Mignemi, "Classical dynamics on curved Snyder space," Classical and Quantum Gravity, vol. 31, no. 10, Article ID 105010, 2014.

[36] S. Mignemi and R. Štrajn, "Snyder dynamics in a Schwarzschild spacetime," Physical Review D, vol. 90, no. 4, Article ID 044019, 2014.
[37] M. M. Stetsko, "Dirac oscillator and nonrelativistic Snyder-de SITter algebra," Journal of Mathematical Physics, vol. 56, no. 1, Article ID 012101, 17 pages, 2015.

[38] G. Amelino-Camelia, A. Marcianò, M. Matassa, and G. Rosati, "Deformed Lorentz symmetry and relative locality in a curved/ expanding spacetime," Physical Review D-Particles, Fields, Gravitation and Cosmology, vol. 86, no. 12, Article ID 124035, 2012.

[39] J. Kowalski-Glikman and G. Rosati, "Relative locality in curved spacetime," Modern Physics Letters A, vol. 28, no. 22, Article ID 1350101, 7 pages, 2013.

[40] C. N. Yang, “On quantized space-time," APS Journals Archive, vol. 72, no. 9, p. 874, 1947.

[41] R. Gilmore, Lie Groups, Lie Algebras and Some of Their Applications, John Wiley \& Sons, 1974.

[42] P. A. M. Dirac, Lectures on Quantum Mechanics, Yeoshua University, New York, NY, USA, 1964. 

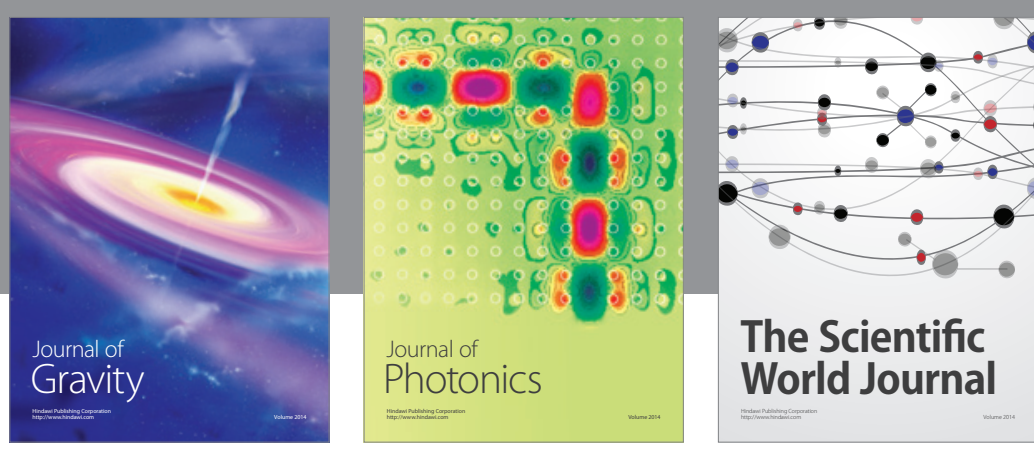

The Scientific World Journal
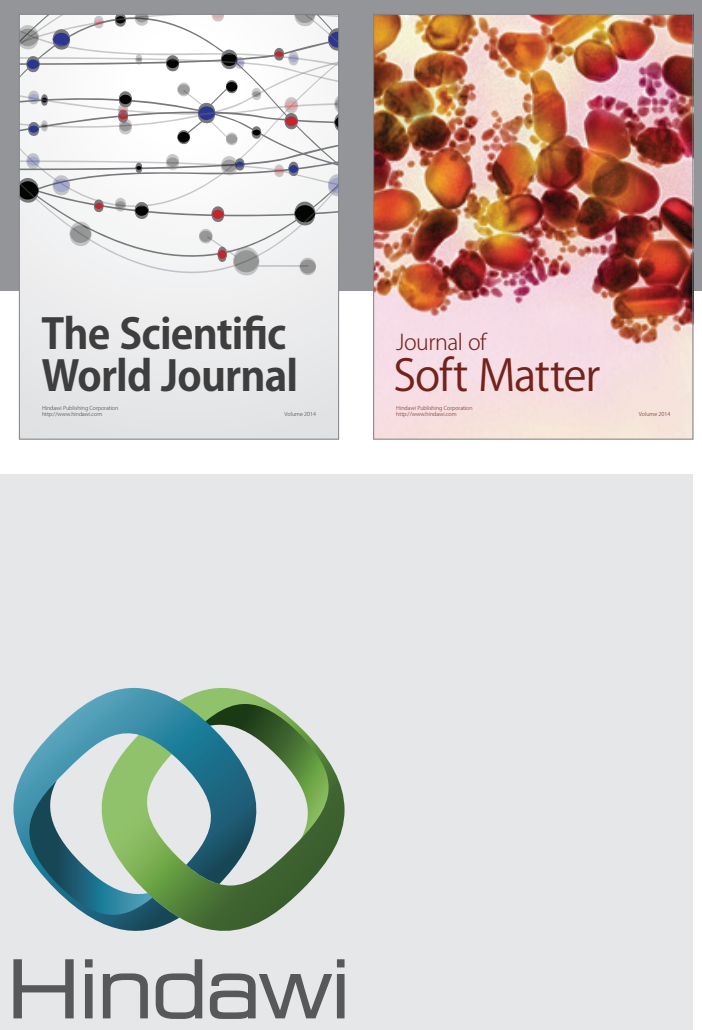

Submit your manuscripts at

http://www.hindawi.com

nternational Journal of

Statistical Mechanics
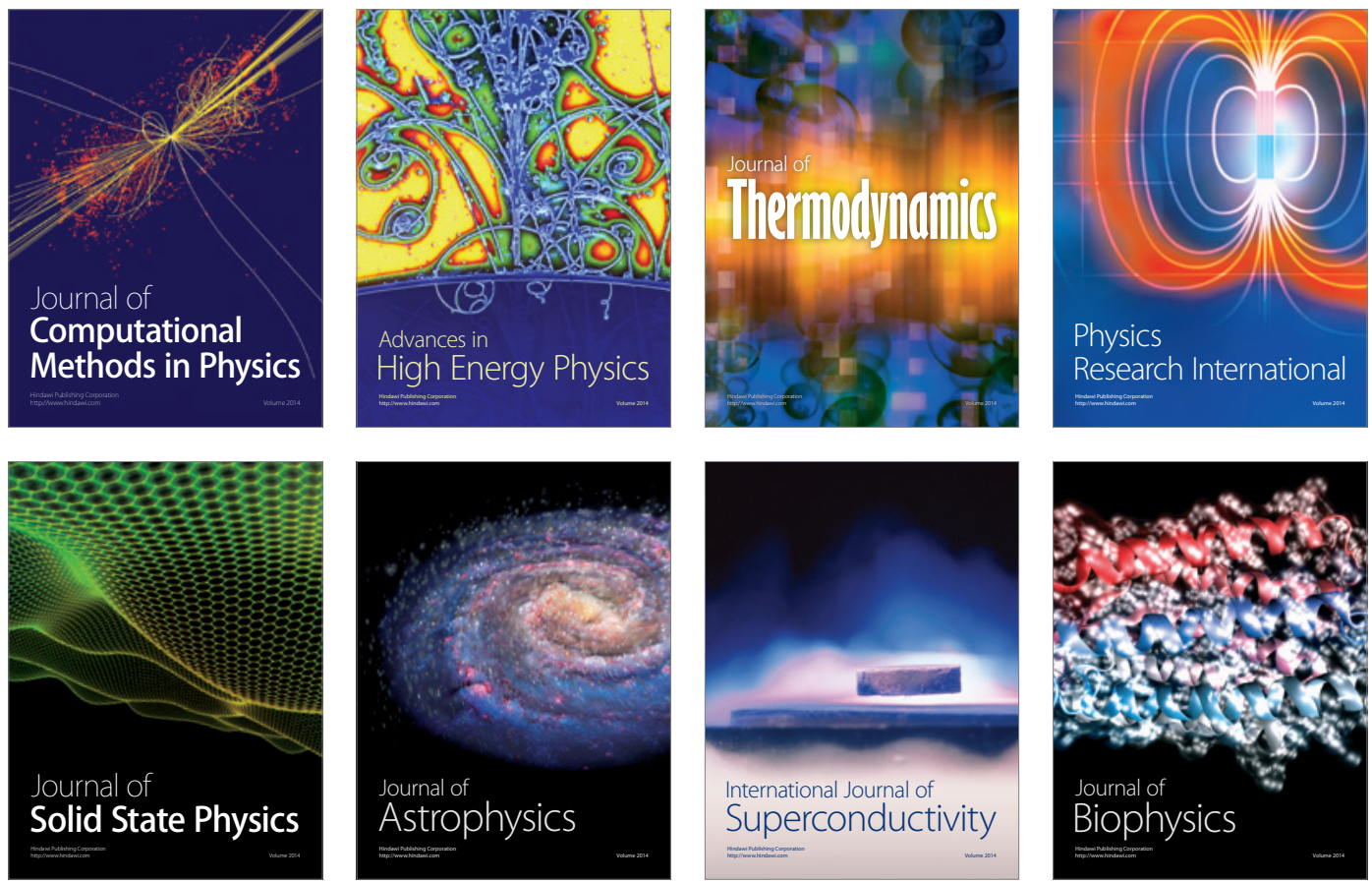
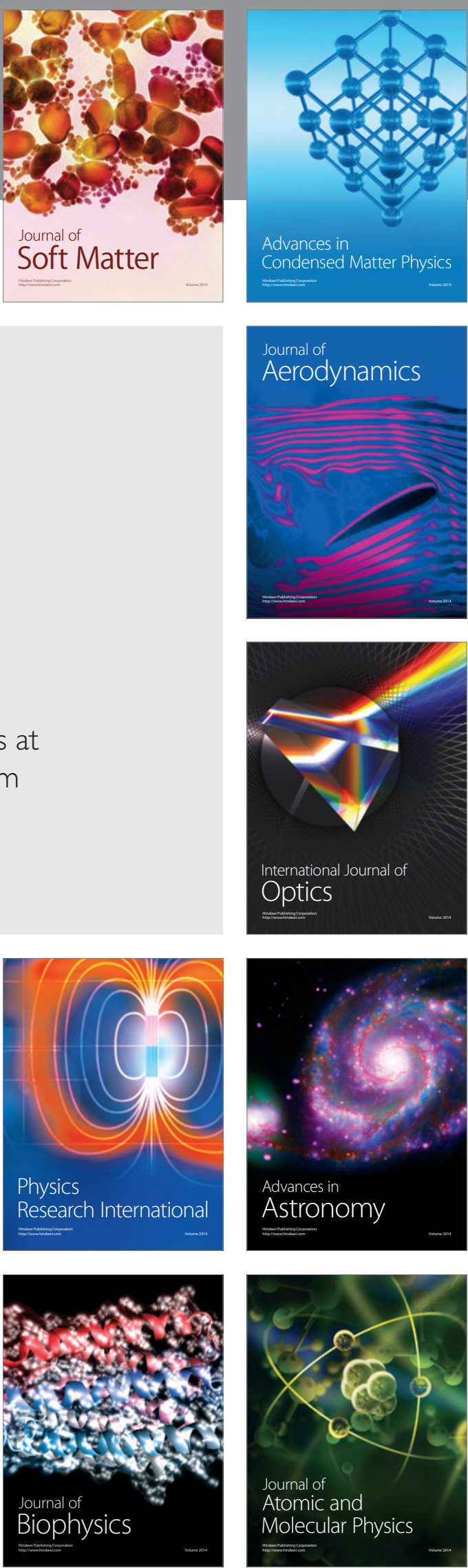